DOI: https://doi.org/10.46296/rc.v3i6.0017

\title{
Sistema de gestión de inocuidad alimentaria y la calidad en empresas pesqueras
}

\section{Food safety and quality management system in fisheries companies}

\author{
Autor: Soledispa-Lucas Fausto Freddy \\ Universidad Laica Eloy Alfaro de Manabí (ULEAM). Manta, Ecuador. \\ fausto.soledispa@uleam.edu.ec
}

\begin{abstract}
RESUMEN
Una economía que basa su desarrollo en el sector pesquero industrial tiene en las unidades empresariales dedicadas al procesamiento y conservas de atún en el eje principal y aglutinador de las actividades productivas. Se constituye en un encadenamiento que genera valor agregado, se propicia empleo de mano de obra calificada y no calificada, orientando hacia una dinámica que se inserta de manera favorable hacia la matriz productiva con enlaces adecuados hacia una matriz energética, denotando eficacia en el manejo de costos de producción. La producción y riqueza generada impulsan el logro de una economía de bienestar, convirtiéndose en la base del crecimiento económico y desarrollo de la región y el país. La investigación tiene como objetivo principal medir como la calidad tiene un impacto en la competitividad del sector industrial de las empresas enlatadoras de atún en la ciudad de Manta. En tal virtud, la aplicación de la norma de inocuidad alimentaria, constituyen una base crítica para garantizar el cumplimiento de estándares de calidad, como mejora continua en los procesos productivos de la empresa. El estudio analiza otras características con la finalidad de identificar elementos que crean la competitividad de las organizaciones industriales en los mercados. El estudio desarrolló la prueba estadística de Cronbach, obteniendo un alfa de 0,947 , que confiere un grado de fidelidad en los datos recogidos. Los resultados estadísticos revelan que la calidad influye en la competitividad de las empresas del sector industrial pesquero.
\end{abstract}

Palabras claves: Calidad; competitividad; inocuidad alimentaria, enlatados de atún, industria.

\section{ABSTRACT}

An economy that bases its development on the industrial fishing sector, has in the business units dedicated to the processing and canning of tuna in the main axis and unifying of the productive activities. It is constituted in a chain that generates added value, the employment of qualified and unskilled labor is promoted, directing towards a dynamic that is inserted favorably towards the productive matrix with adequate links 
towards an energy matrix, denoting efficiency in the management of production costs. The production and wealth generated drive the achievement of a welfare economy, becoming the basis for economic growth and development in the region and the country. The main objective of the research is to measure how quality has an impact on the competitiveness of the industrial sector of tuna canning companies in the city of Manta. As such, the application of food safety standards constitutes a critical basis for ensuring compliance with quality standards, such as continuous improvement in the company's production processes. The study analyzes other characteristics in order to identify elements that create the competitiveness of industrial organizations in the markets. The study developed the Cronbach statistical test, obtaining an alpha of 0.947 , which confers a degree of fidelity in the data collected. The statistical results reveal that quality influences the competitiveness of companies in the fishing industry.

Keywords: Quality; competitiveness; food safety, canned tuna, industry.

\section{INTRODUCCIÓN}

La ciudad de Manta, según información estadística revelada en el censo de población y vivienda de 2010, cuenta con 226 mil habitantes, y es una de las ciudades que cuenta con mayor crecimiento y nivel de industrialización en la Provincia de Manabí. Dentro de su territorio se asienta un clúster de empresas relacionadas a la producción de atún enlatado, que se dirige al mercado interno y externo. Cuenta con otros sectores industriales que aportan al desarrollo local como el de grasas y aceites, de pesca fresca, y otros más. Los sectores señalados se apoyan para su mejor operatividad por centros universitarios, instituciones financieras, servicios básicos, vialidad descongestionante, organismos del gobierno, tecnología y comunicaciones, entre otras.

En este contexto se puede evidenciar que, a pesar de representar ventajas para el desarrollo de la ciudad, aun se puede apreciar un nivel insuficiente de competitividad de sus empresas y un elemento que incide directamente es la calidad de sus productos. Esta situación que presentan los bienes y servicios preocupa al empresario, lo que dificulta la permanencia y participación en el mercado, por tanto, quienes se desentienden de asegurar la calidad en sus procesos y productos dejan pasar oportunidades de negocios, de allí que las enlatadoras de atún de la ciudad de Manta requieren gestionar la calidad como condición indispensable para fortalecer sus capacidades productivas. 
Esta situación incide en la pérdida de mercado local, nacional y sobretodo el internacional, puesto que otras naciones desplazan nuestra producción, lo que demanda la preocupación de las empresas para buscar estrategias que permitan superar las dificultades que se les presentan en este mundo de cambios imprevistos. Añadir a la calidad, también requiere la aplicación de un sistema de gestión de seguridad alimentaria, así como establecer Normas Mundiales de Seguridad Alimentaria para la fabricación de alimentos procesados y la preparación de productos primarios suministrados como productos alimentarios, para alcanzar una certificación válida para los productos que hayan sido fabricados o preparados en centros auditados, incluyendo las instalaciones de almacenamientos que estén bajo control directo de la gestión del centro de producción.

Por lo tanto, el estudio considera el planteamiento del problema en términos de ¿cómo la calidad en la competitividad en las empresas enlatadoras de atún de la ciudad de Manta-Ecuador? Así, dentro de este escenario, el aporte de la investigación se orientó a propiciar el mejoramiento de las condiciones productivas y administrativas de las empresas del sector industrial de la pesca, identificando y mejorando los niveles de calidad y competitividad en sus procesos y productos, a partir de establecer sistemas y normas de seguridad alimentarios, que garanticen la inocuidad de los alimentos procesados en toda la cadena alimentaria. En esa dirección, el objetivo del estudio estuvo centrado en determinar el nivel de influencia de la calidad en la competitividad de las empresas enlatadoras de atún.

\section{DESARROLLO}

Con el decurrir del tiempo surgen los paradigmas de la calidad con el fin de orientar la administración y producción de las diversas organizaciones, mismos que surgen a partir del siglo pasado, y que también son aplicados en la industria ecuatoriana. En el caso de la industria atunera de Manta, se apoyan en el conocido modelo europeo el Total Quality Management (TQM) que aplica la metodología de trabajo recomendada para garantizar óptimos resultados en procesos y productos. Así pues, un modelo de referencia para la organización y 
gestión de una empresa permite establecer un enfoque y un marco objetivo, riguroso y estructurado para el diagnóstico de la organización, así como determinar las líneas de mejora continua hacia las cuales deben orientarse los esfuerzos de esta. Por tanto, un modelo de gestión de calidad es un referente permanente y un instrumento eficaz en el proceso de toda organización que, de manera responsable, busca mejorar su cadena productiva.

En lo que respecta al cuidado de la inocuidad de alimentos, se debe anotar que Ecuador es un país que se destaca a nivel mundial por su industria pesquera, particularmente por su sector atunero, que posee la mayor flota de cerco y flota industrial en el Océano Pacífico Oriental, siendo su captura principal el atún y teniendo como industria un gran potencial y capacidad de procesamiento. En este contexto, las empresas dedicadas a la transformación del atún promueven la certificación en las diferentes normas de calidad e inocuidad que se tiene a nivel mundial y dentro del sector alimentario, así como de sostenibilidad y sustentabilidad del recurso marino. En tal sentido, para cumplir con los objetivos las organizaciones industriales atuneras están inmersas en un proceso de mejora continua, lo que significa lograr el mayor nivel de calidad, cumpliendo con altos estándares de calidad, inocuidad, servicio y competitividad que el mercado exige.

Para llegar con la producción a los grandes bloques comerciales como Estados Unidos y la Unión Europea, se aplican estrategias para garantizar y controlar la seguridad alimentaria a lo largo de toda la cadena productiva, que incluye a la cadena logística y de distribución. Los recursos dirigidos hacia la mejora continua han proporcionado como fruto la consecución y mantenimiento, a lo largo de los años, de las más significativas certificaciones en materia de calidad, seguridad alimentaria y sostenibilidad de los recursos naturales. En Manta se encuentra el clúster atunero que incorpora al conjunto de empresas directamente relacionadas con los procesos de captura, procesamiento y comercialización del atún atendiendo a la demanda del mercado.

Para la industria atunera, el sistema de Análisis de Riesgo y Control de Puntos Críticos (HACCP) es una de las principales exigencias para la exportación de productos alimenticios que demanda la Unión Europea, uno de 
los principales mercados que tiene la industria atunera. El sistema está basado en el supuesto de los peligros de seguridad y calidad que pueden existir en diversos puntos de la cadena productiva, desde la captura hasta el consumo, al tomar medidas sistemáticas para evitar el deterioro y la contaminación del atún. Para garantizar el cumplimiento del sistema HACCP es condición necesaria aplicar las Buenas Prácticas de Manufacturas (BPM) y Procedimientos Operativos Estandarizados de Saneamiento (POES), que se constituyen en las herramientas que posibilitan que todos los factores que influyen en la seguridad de los productos sean controlados de manera adecuada. Desde que las naves son acoderadas en el puerto, los expertos en control de calidad certifican que la pesca cumple con los estándares de calidad internacional, por lo que se puede anotar que la industria alimentaria del atún en Manta satisface las exigencias del mercado internacional.

En el contexto en que se desarrolla el sector atunero, la calidad se constituye en parte de la filosofía del trabajo en la industria alimentaria local, puesto que, con el cumplimiento de la normativa internacional los productos reciben un adecuado control de calidad durante las etapas del proceso. Es por tanto, parte de la cultura organizacional que el sector industrial propicie la búsqueda de la calidad como un elemento sustancial para su crecimiento y proyección hacia los mercados internacionales, lo cual no se limita a un resultado técnico asociado solo con la producción, sino que también se relaciona con las exigencias del cliente interno, asimismo comprende la regulación o normativa que rige en los países compradores, procurando el menor impacto negativo al ecosistema y la salud personal.

A partir del año 1999, la inocuidad alimentaria y de alimentos ha sido un tema primordial en la agenda internacional desde el punto de vista político y comercial debido a los serios incidentes acontecidos en el sector alimenticio. Debido a estos problemas están comprometidas a demostrar que las empresas atuneras cuentan con un sistema de inocuidad alimenticio, lo que se convierte en un prerrequisito básico. En tal sentido las empresas atuneras de Manta en la mejora de la calidad y la inocuidad de los alimentos industrializados inician su proceso con la aplicación de BPM, con la cual minimizan los riesgos en los 
alimentos sobre la contaminación tomando muy en cuenta cada uno de los pasos de la cadena agroalimentaria, desde la producción primaria hasta el consumo final.

El conjunto empresarial incorpora, para el mantenimiento general de la inocuidad de los alimentos, los POES que se constituyen en prácticas y procedimientos de satinización puesto que, al producir los alimentos se los implementa para prevenir la contaminación directa de los alimentos producidos, lo cual contribuye a actuar preventivamente en todas las etapas de elaboración de los enlatados de atún y son indispensables para la aplicación de la BPM.

\subsection{La industria atunera y el Sistema de Gestión de Inocuidad Alimentaria}

Los sistemas de inocuidad alimentaria varían de acuerdo con el alcance, estructura, criterios, procesos de certificación y validez. Algunos esquemas tienen mayor aceptación en algunas regiones en el mundo y otros son más empleados o exigidos a los proveedores por empresas transnacionales, mayoristas o minoristas a nivel Mundial. A partir del año 2000 surge la Global Food Satandard Iniciative, en español, la Iniciativa de Seguridad Alimentaria Global (GFSI) con el fin de establecer el reconocimiento de la equivalencia de estándares. Este organismo reúne a los principales actores de la industria alimentaria para impulsar de forma colaborativa la mejora continua en los sistemas de seguridad alimentaria alrededor del mundo. Los líderes de la industria crearon el GFSI con el fin de especificar los requerimientos para el reconocimiento de los programas de certificación definidos en seguridad alimentaria en sus solicitudes de evaluación comparativa. En el GFSI están minoristas, fabricantes y operadores de servicios alimentarios impulsados por la industria, organización que es administradora de comercio llamada "El Foro de Bienes de Consumo" (CGF por sus siglas en inglés).

La GFSI participa con un rol fundamental en la certificación del sistema de inocuidad alimentaria, reconociendo y utilizando como punto referencial a distintos estándares existentes sobre seguridad alimentaria. Cuando un sistema es calificado como punto referencial, significa que el estándar es aceptado por las empresas que necesitan de una norma de este organismo. EI GFSI pretende 
contribuir con la problemática de la seguridad alimentaria identificando a todos los esquemas de certificación y evaluándolos para determinar si cumplen con los elementos mínimos y clarificar el alcance. Esto es de suma importancia puesto que si bien no son iguales si son equivalentes y permiten demostrar conformidad con un sistema de gestión de inocuidad alimentario.

\subsection{Los esquemas de inocuidad alimentaria reconocidos por el GFSI}

Actualmente, se cuenta con una gran cantidad de esquemas que han sido sometidos a evaluación, comparación y aprobación por la GFSI. Todos ellos presentan características distintas en relación tanto con el alcance, los criterios estudiados y analizados, su estructura, la validez y la manera en cómo se presenta. Así los esquemas reconocidos por este organismo mundial son:

a) BRC Global Standards: Global BRC (British Retil Consortiun)

b) IFS Internacional Featured Standars

c) The Global Aquaculture Alliance

d) FSSC 22000 ó FS 22000 (Food Safety System Certification)

e) SQF Institute: SQF 2000 (Safe Quality Food)

f) Primus GFS

g) GRMS (Global Red Meat Standar)

h) CANADA GAP (On Farm Food Safety For Fresh Fruit and Vegetables)

i) GLOBAL G.A.P.

Los esquemas establecidos por la GFSI cumplen satisfactoriamente los requisitos establecidos, sin embargo, cada esquema emplea un enfoque específico.

\section{METODOLOGÍA}

El estudio se circunscribe como investigación de tipo descriptivo. Según Hernández, (2014), "con los estudios descriptivos se busca especificar las 
propiedades, características y los perfiles de personas, grupos, comunidades, procesos, objetos o cualquier otro fenómeno que se someta a un análisis". Con la información recogida, se realizó la descripción, el análisis y la interpretación de manera sistemática de las características manifestadas del fenómeno investigado con respecto a la realidad del escenario planteado. Fairlie, (2012).

Una investigación de tipo correlacional, siguiendo a Hernández, (2014), "tiene como finalidad conocer la relación o grado de asociación que existe entre dos o más conceptos, categorías o variables en una muestra o contextos particulares. Para realizar la evaluación del grado de asociación entre dos o más variables, en los estudios correlaciónales primero se mide cada una de éstas, y después se cuantifican, analizan y establecen vinculaciones". También es de tipo no experimental, porque no se manipularán deliberadamente las variables. De acuerdo a la evolución del acontecimiento es un estudio transversal, en cuanto los datos han sido tomados en un tiempo delimitado y una población en un momento determinado, lo que ha permitido arribar a conclusiones acerca del fenómeno.

Finalmente, se considera de tipo cuantitativa, debido a que utilizó procedimientos para la medición de las variables en términos cuantitativos, la que se expresa en forma de un instrumento de investigación. La población está constituida por todas las empresas clasificadas en el sector, que en este caso son 10. En la Tabla 1, se presentan los nombres de las empresas consideradas.

Tabla 1. Empresas enlatadoras de atún en la ciudad de Manta

\begin{tabular}{|c|l|l|l|}
\hline$\#$ & \multicolumn{1}{|c|}{ RAZON SOCIAL } & \multicolumn{1}{c|}{ SECTOR INDUSTRIAL } & \multicolumn{1}{c|}{ DIRECCIÓN MANTA } \\
\hline 1 & $\begin{array}{l}\text { CONSERVAS ISABEL } \\
\text { ECUATORIANA S.A. }\end{array}$ & $\begin{array}{l}\text { Procesamiento y conservas } \\
\text { de pescados y sardinas }\end{array}$ & Calle 125 y Av. 103 \\
\hline 2 & $\begin{array}{l}\text { INDUSTRIA ECUATORIANA } \\
\text { PRODUCTORA DE ALIMENTOS } \\
\text { C.A. INEPACA }\end{array}$ & $\begin{array}{l}\text { Procesamiento y conservas } \\
\text { de pescados y sardinas }\end{array}$ & $\begin{array}{l}\text { Malecón S/N Barrio } \\
\text { Miraflores }\end{array}$ \\
\hline 3 & $\begin{array}{l}\text { SOCIEDAD ECUATORIANA DE } \\
\text { ALIMENTOS Y FRIGORIFICOS }\end{array}$ & $\begin{array}{l}\text { Procesamiento y conservas } \\
\text { de pescados y otros } \\
\text { productos marinos }\end{array}$ & $\begin{array}{l}\text { Malecón de Los } \\
\text { Esteros, Calle 124 }\end{array}$ \\
\hline 4 & $\begin{array}{l}\text { MARBELIZE S.A. } . \text { SEAFMAN } \\
\text { MANTAcesamiento y conservas } \\
\text { de pescados }\end{array}$ & $\begin{array}{l}\text { Km 5 1/2 Vía Manta } \\
\text { Rocafuerte }\end{array}$ \\
\hline 5 & EUROFISH S.A. & $\begin{array}{l}\text { Procesamiento y conservas } \\
\text { de pescados }\end{array}$ & $\begin{array}{l}\text { Cdla. La Pradera, Urb. } \\
\text { Arroyo Azul }\end{array}$ \\
\hline
\end{tabular}


Revista Científica Arbitrada de Investigación en Comunicación, Marketing y Empresa REICOMUNICAR. Vol. 3, Núm. 6 (jul - dic 2020) ISSN: 2737-6354.

\begin{tabular}{|l|l|l|l|}
\hline 6 & $\begin{array}{l}\text { PESPACA PESQUERA DEL } \\
\text { PACÍFICO C.A. }\end{array}$ & $\begin{array}{l}\text { Procesamiento y conservas } \\
\text { de pescados }\end{array}$ & $\begin{array}{l}\text { Av. 24 de Mayo y } \\
\text { Calle } 5 \text { (Of) }\end{array}$ \\
\hline 7 & $\begin{array}{l}\text { INDUSTRIAS ENLATADOS } \\
\text { ALIMENTICIOS CIA LTDA. } \\
\text { IDEAL }\end{array}$ & $\begin{array}{l}\text { Procesamiento y conservas } \\
\text { de pescados }\end{array}$ & $\begin{array}{l}\text { Carretera Manta } \\
\text { Portoviejo Km } 71 \frac{1}{2}\end{array}$ \\
\hline 8 & ASISERVY S.A. & $\begin{array}{l}\text { Procesamiento y elaboración } \\
\text { de pescado y otros productos }\end{array}$ & $\begin{array}{l}\text { Km } 51 / 2 \text { Vía Manta } \\
\text { Rocafuerte }\end{array}$ \\
\hline 9 & FISHCORP. & $\begin{array}{l}\text { Procesamiento y elaboración } \\
\text { de pescado y otros productos } \\
\text { marinos }\end{array}$ & $\begin{array}{l}\text { Carretera Manta } \\
\text { Rocafuerte Km } 41 / 2\end{array}$ \\
\hline 10 & $\begin{array}{l}\text { TÉCNICA Y COMERCIO DE LA } \\
\text { PESCA TECOPESCA C.A. }\end{array}$ & $\begin{array}{l}\text { Procesamiento de pescado y } \\
\text { otros productos marinos }\end{array}$ & $\begin{array}{l}\text { Km } 41 / 2 \text { Vía Manta } \\
\text { Rocafuerte }\end{array}$ \\
\hline
\end{tabular}

Fuente: Subsecretaría de Agricultura, Ganadería, Acuacultura y Pesca.

Elaboración: Autor.

El estudio considera una población finita, puesto que las empresas que participan en el sector industrial son de accesible observación. De acuerdo con el criterio de expertos, cuando una población es menor a 50 individuos, se puede asimilar a la muestra. Por ello en estas situaciones es más ventajoso inspeccionar todos los elementos de una población (Stevenson, 2001) De allí que, la muestra seleccionada corresponde al total de las empresas del sector industrial de la pesca. Para elaborar el diseño del instrumento de medición se revisó la literatura correspondiente observando cada una de las variables, efectuadas bajo la metodología Likert, la cual considera una escala que va del 1 al 5, en donde el 1 significa el valor más bajo, y el 5 representa el valor más alto. La encuesta tuvo una ficha de opinión de expertos, calificando el instrumento de excelente. Para realizar la fiabilidad del instrumento, se empleó un análisis de confiabilidad proveniente de los resultados de la aplicación de la encuesta. El coeficiente considerado es el Alfa de Cronbach.

\section{RESULTADOS}

\subsection{Esquemas de calidad e inocuidad de productos enlatados en la industria del atún en Manta.}

Las empresas industriales que fabrican los enlatados de atún en la ciudad 
de Manta tienen establecidos sus Sistemas de Gestión de Inocuidad Alimentaria (SGIA) con su propio alcance, estructura, criterios, procesos de certificación y validez. Entre los esquemas de inocuidad alimentaria está el de Análisis de Riesgos y Puntos Críticos de Control HACCP, mediante el cual la seguridad alimentaria se cumple con el análisis y control de los peligros físicos, químicos y biológicos en los procesos de adquisición de materia prima, abastecimiento, manufactura, la distribución y el consumo del producto terminado. Las condiciones previas para la aplicación son las Buenas Prácticas de Manufacturas (BPM), que son una serie de prácticas y procedimientos claves para lograr la inocuidad de los alimentos que se manipulan, puesto que la inocuidad es una responsabilidad de todos los trabajadores que participan en la cadena alimentaria. Otro prerrequisito básico de las HACCP son los POES que contribuye a actuar preventivamente en todas las etapas de elaboración del atún enlatado.

Las empresas atuneras emplean también la norma de seguridad alimentaria BRC Global, que está inserta en la GFSI, cuyo fin es certificar la gestión de la calidad, con la cual se protege a los productos alimenticios en el cumplimiento de las obligaciones legales de seguridad alimentaria garantizando la protección del consumidor. La IFS es la otra norma de auditoría de calidad y seguridad alimentaria de productos alimenticios que se enfoca en la seguridad del consumo de alimentos, cuando existen riesgos de contaminación de los productos en empaque garantizando una calidad acorde a las especificaciones de los clientes, mejorando continuamente los procesos de trabajo, siendo empleada en las empresas del sector atunero de Manta.

También las industrializadoras de atún de Manta emplean otras normas tales como la OU KOSHER, que es una certificación de alimentos según códigos y leyes relacionadas a la tradición judía. También emplean la norma denominada GMP (GOOD MANUFACTURINE PRACTICE) que significa que el fabricante cumple la norma correcta de fabricación, es decir que los productos alimenticios se fabrican de forma consistente y se controlan aplicando las normas de calidad acordes con la legislación vigente, con lo que se asegura que los productos son de calidad. Las otras normas aceptadas por la GFSI como las The Global Aquaculture Alliance, FSSC 22000 ó FS 22000 (Food Safety System 
Certification), SQF Institute: SQF 2000 (Safe Quality Food), Primus GFS, GRMS (Global Red Meat Standar), CANADA GAP (On Farm Food Safety For Fresh Fruit and Vegetables) y GLOBAL G.A.P., no las han integrado a su sistema de gestión de inocuidad alimentaria las empresas industriales del sector atunero de Manta.

Sin embargo, no hay que desconocer que la Norma SQF 2000 es la que contempla el más completo conjunto de requisitos en el ámbito de la calidad e inocuidad de los productos alimentarios enlatados, ya sea en procesos de crianza o cultivo, preproceso, proceso y producción. De igual forma la norma "Sistema de Gestión de calidad e inocuidad GMP+B2", las empresas del clúster atunero de Manta no la están aplicando, las cuales indudablemente garantizan la calidad de los productos a los clientes. Los otros esquemas tienen un alcance más limitado, aunque por la naturaleza de pertenecer al sector pesquero se adaptan a las otras normas como BRC ó IFS, con las cuales las industrias atuneras son auditadas anualmente en sus procesos y productos, mediante las cuales propician una mayor flexibilidad para satisfacer las necesidades de los clientes o para hacer frente a los cambios futuros en las normativas existentes.

Las normas de inocuidad alimentaria se encuentran en correspondencia con la norma internacional ISO 22000, la que especifica los requisitos de un Sistema de Gestión de la Inocuidad Alimentaria (SGIA). Con la norma ISO 22000, se hace explícita la aplicación de la norma HACCP, y las demás normas que tratan de la inocuidad alimentaria en cadena de abastecimientos de alimentos y permitiendo una solución específica para las buenas prácticas a nivel global. De allí que los SGIA que cumplen con la ISO 22000 son susceptibles de certificación. El sistema de calidad e inocuidad se articula con las normas ISO 9001: 2008, la cual consiste en varios estándares de calidad en donde se establecen criterios de gestión para un sistema de control de calidad que tiene un componente directo en el cliente, así como motivación de la fuerza laboral, implicación de gerencia y mejoras continuas en la calidad de los productos enlatados. Los principios de la norma ISO propician las herramientas para guiar a las empresas que procuran asegurar la calidad de los productos, cumpliendo de forma consistente con los requerimientos de los clientes. 


\subsection{Normas para la sostenibilidad, sustentabilidad ambiental y comercio seguro}

Para garantizar el cumplimiento de los estándares en el manejo de los recursos naturales las industrias del sector atunero de Manta también cumplen con las normativas que tratan sobre la sostenibilidad y sustentabilidad de los recursos pesqueros, obligatorias a nivel mundial. Una de las imprescindibles es la Marine Stewar Ship Council conocida por sus siglas MSC, la que corresponde a estándares de trazabilidad para la cadena de custodio de productos pesqueros, desde pesquerías certificadas hasta su destino final. Con ella se busca la confianza de los compradores y consumidores que las industrias certificadas por MSC se encuentran autorizadas para procesar productos provenientes de pesquerías que cumplen los estándares medioambientales del MSC para la pesca sostenible. Otra norma es la denominada Dolphin Safe donde los estándares y políticas forman parte del monitoreo para la pesca del atún que busca detener la persecución, pesca y captura internacional de los delfines y ballenas, así como prevenir el tráfico de mamíferos para lograr que los océanos otorguen seguridad para la vida y el medio ambiente.

Así mismo, la industria atunera de Manta cuenta con normas que se relacionan con el comercio seguro a nivel global, entre ellas está la Business Alliance For Secure Commerce, conocida por sus siglas BASC, la que es una alianza comercial empresarial, generada para promover la seguridad en el comercio. Sus políticas están diseñadas para enfrentar el problema mundial del contrabando en el comercio y mejorar significativamente las políticas de seguridad en instituciones gubernamentales y no gubernamentales. La industria atunera también trabaja con la norma Bussines Social Compliance Iniciative cuyas siglas son BSCl, la misma que es una iniciativa de la Asociación de Comercio Exterior (FTA) con el ánimo de alcanzar el "Libre Comercio y Sostenibilidad Comercial", orientando un código de conducta que posibilita a las industrias trabajar dentro de un marco de principios de Responsabilidad Social Corporativa, así como los estándares internacionales de trabajo. La norma del $\mathrm{BSCl}$ realiza un trabajo en conjunto con entidades gubernamentales mediante las cuales las empresas participan, desarrollan e implementan el sistema en mejores condiciones de trabajo, en la cadena de suministro global. 
Otras normas exigidas por diversos países a los que se exporta la producción de enlatados de atún en el cumplimiento de la calidad e inocuidad alimentaria son FDA, Senasa (Argentina), Punto Verde, Europa Unión, Difoa (Brasil), Rossel Khoznadzor Certification (Rusia), Invima, Sello Se Hace Bien. Así mismo el sector industrial de enlatados de atún cumple con los requisitos que le impone el Sistema Ecuatoriano de la Calidad para salvaguardar el cumplimiento de conformidades de los productos y servicios. La institución que tiene la capacidad de ejecutar los principios y mecanismos de la calidad y la evaluación de conformidad es el Ministerio de Industrias y Productividad (MIPRO), conjuntamente con el Instituto Ecuatoriano de Normalización (INEN), el Organismo de Acreditación Ecuatoriano (OAE), ante la cual las industrias atuneras responden por la conformidad de los productos.

\section{CONCLUSIONES Y DISCUSIÓN}

A continuación, se presenta las principales conclusiones de la investigación:

El primer resultado alcanzado revela que existe una influencia, con alto nivel de significancia de la calidad sobre la competitividad en las empresas enlatadoras de atún en la Ciudad de Manta - Ecuador, situación que se asume de manera positiva. El nivel de alfa teórico está por debajo del 0,05.

Se comprobó la existencia de una relación positiva entre el liderazgo y la competitividad del sector industrial de las empresas enlatadoras de atún de la ciudad de Manta - Ecuador. El grado de asociación es aceptable puesto que el nivel de significancia está por debajo del coeficiente 0,05 , la influencia entre las variables es perfecta.

Se comprobó que existe una asociación positiva directa de la Planificación con la Competitividad, en un alto nivel en las empresas enlatadoras de atún de la ciudad de Manta - Ecuador, con lo cual se aceptó la hipótesis alternativa, pues el grado de significancia es menor que 0.05. Se denota que existe una significativa concordancia entre las variables. 
Se demostró que existe una influencia positiva directa en alto grado de la Gestión por Procesos en el nivel de competitividad de las empresas enlatadoras de atún de la ciudad de Manta - Ecuador. El grado de asociación es menor de $0.05 s$.

En relación con los antecedentes de la investigación, se puede ver que existe una correlación positiva entre investigaciones realizadas y el presente estudio. Así, en el trabajo efectuado por Álvarez García, Iñigo (2008), Análisis de implantación de la calidad. Estudio empírico en el sector de alojamiento turístico, manifiesta sobre los beneficios alcanzados al aplicar la calidad en las empresas del sector industrial, tanto en Europa como en Estados Unidos. Se destaca que "la calidad contribuye a mejorar la competitividad de los productos, a mejorar la comunicación, las relaciones entre los empleados, la competitividad, así como la cuota del mercado. Withers y Ebrahimpour, (2001). La calidad es impuesta como un sistema que favorece el mejoramiento de sus procesos, volviéndolas más competitivas. Además, que a partir de las entrevistas estructuradas aplicadas a los empresarios muestran que la calidad se constituye en factor clave fundamental para el sector industrial. Y, el liderazgo convierte a las empresas en referentes a seguir por el conglomerado de las demás empresas instaladas en el sector.

En relación con la investigación desarrollada por Heras Iñaki (2008), “La gestión de la calidad y competitividad de las empresas de CAPV' establece que las empresas españolas tienen las mayores intensidades de certificaciones de calidad, a nivel europeo, lo que les otorga ventajas competitivas ante sus competidoras. Los procesos se hacen de mejor manera, la productividad se incrementa, el compromiso laboral es más intenso, el liderazgo tiene una mayor incidencia en las decisiones gerenciales. La calidad tanto en el ámbito profesional como académico es considerada como uno de los factores de éxito competitivo, el más importante. La calidad se constituye en un soporte básico para el desempeño de las empresas en cualquier país. Así, también resulta en el conglomerado industrial de conservas de atún en la ciudad de Manta.

De acuerdo con el análisis cuantitativo las características comunes entre las empresas enlatadoras de atún de la ciudad de Manta, es que consiguen 
resultados exitosos en los mercados y que los gerentes dirigen activamente el programa de calidad, permanentemente revisando su eficacia. Los empleados se identifican con los valores empresariales y apoyan la implantación de cambios en la organización. Respecto a la planificación, los gerentes comunican la estrategia y objetivos al personal para cumplir con lo establecido por la empresa. Además, identifican y desarrollan procesos claves a partir de la estrategia empresarial. En lo relacionado a la gestión por procesos se realiza el control y la mejora continua de los procesos claves de la organización. Las empresas se preocupan de introducir la mejora en los productos para llegar a nuevos mercados, así como la conducta de anticiparse a las necesidades del mercado.

\section{REFERENCIAS}

Álvarez, I. (2008). Análisis de implantación de la calidad. Estudio empírico en el sector de alojamiento turístico, Madrid, España.

Arispe, I., Tapia, M. (2007). Innocuidad y calidad: requisitos indispensables para la protección de la salud de los consumidores. https://www.redalyc.org/pdf/1992/199216580008.pdf.

Bateman, Thomas S. \& Snell, Scott A., (2009). Administración: liderazgo y colaboración en un mundo competitivo, Octava Edición, Ed. McGraw Hill, México.

Berkowitz,

D. (2012).

Industria

Alimentaria.

https://books.google.com.ec/books/about/Cap\%C3\%ADtulo_67_Industria _alimentaria.html?id=fNjftwEACAAJ\&redir_esc=y.

Berumen A., Sergio, \& Sommer Palacios, Octavio (2009), Competitividad, Cluster e Innovación, Ed. McGraw Hill, México.

Carro, R, \& González, D. (2015). Normas HCCP. Sistema de análisis de riesgos y puntos críticos de control, Universidad Nacional de Mar del Plata, Argentina.

Ecuador Ministerio de economía y finanzas, Agenda de competitividad 20122013, http://www.mef.gob.pe/...conpetitividad_2012_2013.pdf. 
Fairlie, E. (2012). El valor de la marca como estrategia para incrementar la capacidad emprendedora en estudiantes de Universidades Nacionales del área de Ciencias Empresariales, Lima, Perú.

Heras, I., ett. All. (2010). Impactos competitivos de las herramientas para la gestión de la calidad, Universidad del país Vasco, España.

Heras, I., ett. All. (2010). La gestión de la calidad y competitividad de las empresas de CAPV, San Sebastián, España.

Hernández Sampieri, R., ett. all. (2014). Metodología de la investigación, México.

Ministerio de Coordinación de la Producción, Empleo y Competitividad (2011). Agenda para la transformación productiva territorial, Provincia de Manabí.

Organismo Internacional Regional de Sanidad Agropecuaria. (2016). Manual de análisis de peligros y puntos críticos de control, San Salvador.

Organización de las Naciones Unidas para la Agricultura y la Alimentación. (2002). Sistemas de calidad e inocuidad de los alimentos. https://www.who.int/foodsafety/publications/consumer/manual_keys_es.p df?ua=1.

Organización Mundial de la Salud. (2007). Manual sobre las cinco claves para la inocuidad de los alimentos. https://www.who.int/foodsafety/publications/consumer/manual_keys_es.p df?ua=1. 